\title{
Lissajous Orbits at the Collinear Libration Points in the Restricted Three-Body Problem with Oblateness
}

\author{
${ }^{1}$ Astronomy and Meteorology Department, Faculty of Science, Al-Azhar University, Cairo, Egypt \\ ${ }^{2}$ Mathematics Department, Faculty of Science (Girls), Al-Azhar University, Cairo, Egypt \\ ${ }^{3}$ Mathematics Department, Faculty of Science (Girls), Al Qassim University, Buraidah, Saudi Arabia \\ Email: ahmed_hafez@azhar.edu.eg,mnader_is@azhar.edu.eg,d.sahar92@azhar.eg
}

Ahmed H. Ibrahim1, Mohammed N. Ismail'1, Afaf S. Zaghrout'², Saher H. Younis ${ }^{2}$, Mayadah 0. El Shikh ${ }^{3}$

How to cite this paper: Ibrahim, A.H. Ismail, M.N., Zaghrout, A.S., Younis, S.H. and El Shikh, M.O. (2018) Lissajous Orbits at the Collinear Libration Points in the Restricted Three-Body Problem with Oblateness. World Journal of Mechanics, 8, 242-252.

https://doi.org/10.4236/wjm.2018.86020

Received: May 13, 2018

Accepted: June 23, 2018

Published: June 26, 2018

Copyright $(9) 2018$ by authors and Scientific Research Publishing Inc. This work is licensed under the Creative Commons Attribution International License (CC BY 4.0).

http://creativecommons.org/licenses/by/4.0/

\begin{abstract}
In the present work, the collinear equilibrium points of the restricted three-body problem are studied under the effect of oblateness of the bigger primary using an analytical and numerical approach. The periodic orbits around these points are investigated for the Earth-Moon system. The Lissajous orbits and the phase spaces are obtained under the effect of oblateness.
\end{abstract}

\section{Keywords}

Lissajous Orbits, Periodic Orbits, Libration Points, Restricted Three-Body Problem, Oblate Spheroid

\section{Introduction}

One of the most important object in astrodynamics is the restricted three-body problem, which has many applications in space missions. It deals with the motion of an infinitesimal mass under the effect of gravitational attraction of two bodies, called primaries, which move in a Keplerian orbit around their common center of mass.

This problem has five libration points: three of them are called the collinear points $L_{1}, L_{2}$ and $L_{3}$, which lie on the line joining the two primaries; the other two are called the triangular points $L_{4}$ and $L_{5}$ [1] [2]. Several studies have been carried out on the collinear libration points by considering the oblateness of one or two primaries for the circular restricted three-body problem. Sharma and Subbarao [3] investigated the the locations of the five libration points under the effect of oblateness of the more massive primary for some systems of celestial 
bodies. Ibrahim A. [4] investigated the libration points for the Sun-Earth-Moon System. Ismail M. [5] studied the effect of solar radiation pressure on the libration points of the restricted four-body problem.

The existence of the periodic orbits near the collinear libration points was treated by many authors. Grgory A. [6] determined a class of Eight Lissajous orbits near collinear libration points by using Lindstedt Poincares technique. Celletti A. [7] analyzed the Lissajous and halo orbits near the collinear libration points by using the classical perturbation theory. In this paper, the restricted three-body problem is studied by considering the more massive primary as an oblate spheroid. The Lissajous orbits in this case are presented, and also the phase spaces are obtained.

\section{Equations of Motion}

Using a barycentric-synodic coordinate system $(\xi, \eta, \zeta)$ and dimensionless variables, the equations of motion of a test particle in the circular restricted three-body problem under the effects of oblateness of the bigger primary can be expressed as

$$
\begin{gathered}
\ddot{\xi}-2 n \dot{\eta}=U_{\xi} \\
\ddot{\eta}+2 n \dot{\xi}=U_{\eta} \\
\ddot{\zeta}=U_{\zeta}
\end{gathered}
$$

where,

$$
\begin{gathered}
U_{\xi}=\xi n^{2}-\frac{(1-\mu)(\mu+\xi)}{R_{1}^{3}}-\frac{(A(1-\mu))(\xi-\mu)}{R_{1}^{5}}-\frac{\mu(\mu+\xi-1)}{R_{2}^{3}}, \\
U_{\eta}=n^{2} \eta-\frac{\eta(1-\mu)}{R_{1}^{3}}-\frac{\eta(A(1-\mu))}{R_{1}^{5}}-\frac{\eta \mu}{R_{2}^{3}} \\
U_{\zeta}=-\frac{\zeta(1-\mu)}{R_{1}^{3}}-\frac{\zeta(A(1-\mu))}{R_{1}^{5}}-\frac{\zeta \mu}{R_{2}^{3}}
\end{gathered}
$$

where $R_{1}=\mu+\xi$ and $R_{2}=\mu+\xi-1$.

The mean motion $\mathrm{n}$ of the primaires is given by $n^{2}=1+(3 / 2) A$, where $A=\left(r_{e}^{2}-r_{p}^{2}\right) /\left(5 R^{2}\right)$ is the oblateness coefficient of $m_{1}$ having the equatorial and polar radii as $r_{e}$ and $r_{p}$, respectively.

\section{Location of the Libration Points}

The collinear equilibrium points can be obtained by solving Equations (4), (5) and (6) when $\eta=\zeta=0$ that yields

$$
U_{\xi}=\xi n^{2}-\frac{(1-\mu)(\mu+\xi)}{R_{1}^{3}}-\frac{(A(1-\mu))(\xi-\mu)}{R_{1}^{5}}-\frac{\mu(\mu+\xi-1)}{R_{2}^{3}}
$$

The coordinate of the collinear Points $L_{1}, L_{2}$ and $L_{3}$ are

$$
\xi_{1}=\mu-1-x_{1}, \quad \xi_{2}=\mu-1+x_{2}, \quad \xi_{2}=\mu+x_{3}
$$


where $x_{1}, x_{2}$ and $x_{3}$ satisfy seventh degree polynomials:

$$
\begin{aligned}
& (3 A+2) x_{1}^{7}+(-3 A \mu+15 A-2 \mu+10) x_{1}^{6}+(-12 A \mu+30 A-8 \mu+20) x_{1}^{5} \\
& +(-18 A \mu+30 A-12 \mu+22) x_{1}^{4}+(-12 A \mu+15 A-4 \mu+14) x_{1}^{3} \\
& +(-6 A \mu+6 A+8 \mu+4) x_{1}^{2}+8 \mu x_{1}+2 \mu=0 \\
& (3 A+2) x_{2}^{7}+(3 A \mu-15 A+2 \mu-10) x_{2}^{6}+(-12 A \mu+30 A-8 \mu+20) x_{2}^{5} \\
& +(18 A \mu-30 A+12 \mu-22) x_{2}^{4}+(-12 A \mu+15 A-4 \mu+14) x_{2}^{3} \\
& +(6 A \mu-6 A-8 \mu-4) x_{2}^{2}+8 \mu x_{2}-2 \mu=0 \\
& \quad(3 A+2) x_{3}^{7}+(3 A \mu+6 A+2 \mu+4) x_{3}^{6}+(6 A \mu+3 A+4 \mu+2) x_{3}^{5} \\
& \quad+(3 A \mu+2 \mu-2) x_{3}^{4}+(3 A \mu-3 A+2 \mu-2) x_{3}^{3} \\
& \quad+(6 A \mu-6 A-8 \mu-4) x_{3}^{2}+8 \mu x_{3}-2 \mu=0
\end{aligned}
$$

Each of Equations (8), (9) and (10) has three complex pairs roots, whose equal imaginary parts in magnitude and only one real number represents the position of corresponding collinear point. The intersections of curves for Figure 1 with horizontal axis represent the three collinear libration points under the effect of oblateness.

\section{The Motion around Collinear Libration Points}

To study the motion of an infinitesimal neighborhood around the libration points the variationally variables $(\xi, \eta, \zeta)$ are introduced such that

$$
\xi=x-x L_{i}, \eta=y-y L_{i}, \zeta=z-z L_{i}
$$

where, $x L_{i}, y L_{i}, z L_{i}$ represent the shift around the collinear points.

The resulting linear variationally equations for motion about $L_{i}$ are written as follows,

$$
\begin{aligned}
& \ddot{\xi}-2 n \dot{\eta}=\xi U_{x x}+\eta U_{x y}+\zeta U_{x z}, \\
& \ddot{\eta}+2 n \dot{\xi}=\eta U_{y y}+\zeta U_{y z}+\xi U_{x y},
\end{aligned}
$$

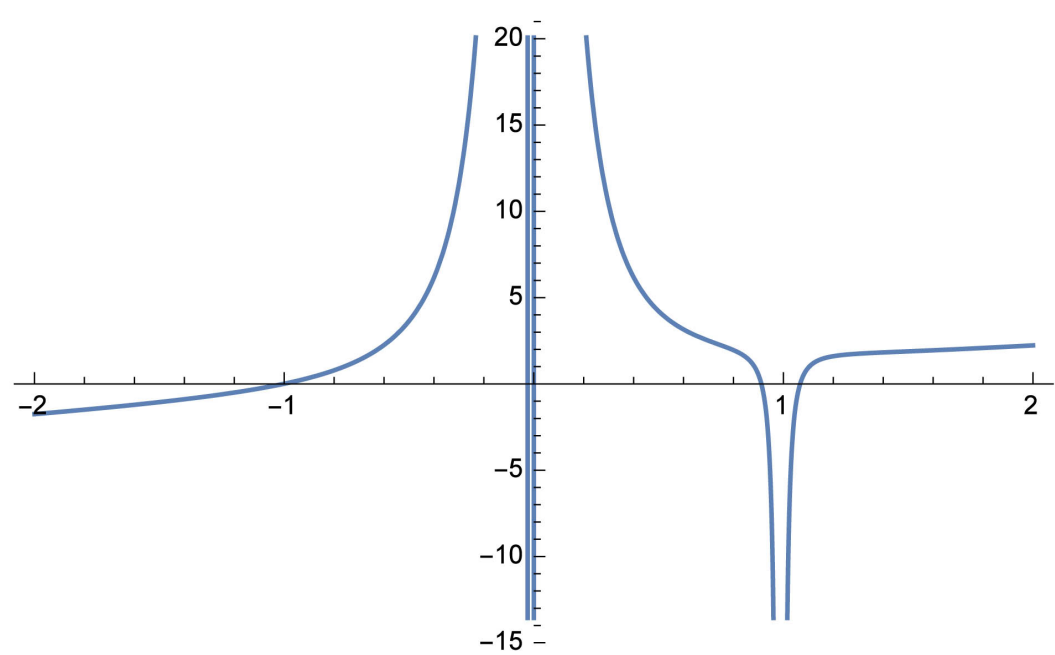

Figure 1. The positions of collinear libration points. 


$$
\ddot{\zeta}=\zeta U_{z z} .
$$

where,

$$
\begin{aligned}
& U_{x x}=1-\frac{3 A(1-\mu)}{2\left((\mu+x)^{2}+y^{2}+z^{2}\right)^{5 / 2}}+\frac{15 A(1-\mu)(\mu+x)^{2}}{2\left((\mu+x)^{2}+y^{2}+z^{2}\right)^{7 / 2}}+\frac{3 A}{2} \\
& -\frac{3 \mu(\mu+x-1)^{2}}{\left((\mu+x-1)^{2}+y^{2}+z^{2}\right)^{5 / 2}}+\frac{\mu}{\left((\mu+x-1)^{2}+y^{2}+z^{2}\right)^{3 / 2}} \\
& -\frac{1-\mu}{\left((\mu+x)^{2}+y^{2}+z^{2}\right)^{3 / 2}}+\frac{3(1-\mu)(\mu+x)^{2}}{\left((\mu+x)^{2}+y^{2}+z^{2}\right)^{5 / 2}} \\
& U_{y y}=1-\frac{15 A(1-\mu) y^{2}}{2\left((\mu+x)^{2}+y^{2}+z^{2}\right)^{7 / 2}}-\frac{3 A(1-\mu)}{2\left((\mu+x)^{2}+y^{2}+z^{2}\right)^{5 / 2}}+\frac{3 A}{2} \\
& +\frac{3 \mu y^{2}}{\left((\mu+x-1)^{2}+y^{2}+z^{2}\right)^{5 / 2}}+\frac{3(1-\mu) y^{2}}{\left((\mu+x)^{2}+y^{2}+z^{2}\right)^{5 / 2}} \\
& -\frac{\mu}{\left((\mu+x-1)^{2}+y^{2}+z^{2}\right)^{3 / 2}}-\frac{1-\mu}{\left((\mu+x)^{2}+y^{2}+z^{2}\right)^{3 / 2}} \\
& U_{z z}=\frac{5 A(1-\mu) z^{2}}{\left((\mu+x)^{2}+y^{2}+z^{2}\right)^{7 / 2}}-\frac{A(1-\mu)}{\left((\mu+x)^{2}+y^{2}+z^{2}\right)^{5 / 2}} \\
& +\frac{3 \mu z^{2}}{\left((\mu+x-1)^{2}+y^{2}+z^{2}\right)^{5 / 2}}+\frac{3(1-\mu) z^{2}}{\left((\mu+x)^{2}+y^{2}+z^{2}\right)^{5 / 2}} \\
& -\frac{\mu}{\left((\mu+x-1)^{2}+y^{2}+z^{2}\right)^{3 / 2}}-\frac{1-\mu}{\left((\mu+x)^{2}+y^{2}+z^{2}\right)^{3 / 2}} \\
& U_{x y}=U_{y x}=\frac{15 A(1-\mu) y(\mu+x)}{2\left((\mu+x)^{2}+y^{2}+z^{2}\right)^{7 / 2}}-\frac{3 \mu y(\mu+x-1)}{\left((\mu+x-1)^{2}+y^{2}+z^{2}\right)^{5 / 2}} \\
& +\frac{3(1-\mu) y(\mu+x)}{\left((\mu+x)^{2}+y^{2}+z^{2}\right)^{5 / 2}} \\
& U_{x z}=U_{z x}=\frac{15 A(1-\mu) z(\mu+x)}{2\left((\mu+x)^{2}+y^{2}+z^{2}\right)^{7 / 2}}-\frac{3 \mu z(\mu+x-1)}{\left((\mu+x-1)^{2}+y^{2}+z^{2}\right)^{5 / 2}} \\
& +\frac{3(1-\mu) z(\mu+x)}{\left((\mu+x)^{2}+y^{2}+z^{2}\right)^{5 / 2}} \\
& U_{y z}=U_{z y}=\frac{15 A(1-\mu) y z}{2\left((\mu+x)^{2}+y^{2}+z^{2}\right)^{7 / 2}}+\frac{3(1-\mu) y z}{\left((\mu+x)^{2}+y^{2}+z^{2}\right)^{5 / 2}} \\
& +\frac{3 \mu y z}{\left((\mu+x-1)^{2}+y^{2}+z^{2}\right)^{5 / 2}}
\end{aligned}
$$


Because of all the libration points are in-plane, the partial derivatives containing z-components are vanished. Therefore, Equations (11) through (13) become

$$
\begin{aligned}
& \ddot{\xi}-2 n \dot{\eta}=\xi U_{x x}+\eta U_{x y} \\
& \ddot{\eta}+2 n \dot{\xi}=\eta U_{y y}+\xi U_{x y}
\end{aligned}
$$

Then characteristic equation corresponding to Equations (14) and (15) is [8]

$$
\lambda^{4}+\left(\lambda\left(-U_{x x}-U_{y y}+4 n^{2}\right)\right) \lambda^{2}+U_{x x} U_{y y}-U_{x y}^{2}=0
$$

At the collinear points the values of $U_{x x}>0, U_{y y}<0$ and $U_{x y}=0$ hence $U_{x x} U_{y y}-U_{x y}^{2}<0$ so the roots of the characteristic Equation (16) are found to be $\lambda_{1,2}= \pm \lambda, \quad \lambda_{3,4}= \pm i s, \lambda$ and $s$ are real. Let the variational of elements depend on time given by

$$
\begin{aligned}
& \xi=A_{i} \sum_{i=1}^{4} \mathrm{e}^{\lambda_{i} t} \\
& \eta=\beta_{i} \sum_{i=1}^{4} \mathrm{e}^{t \lambda_{i}}
\end{aligned}
$$
where $A_{i}$ and $\beta_{i}$ are constant coefficients. and $\beta_{i}=\frac{A_{i}\left(\lambda_{i}^{2}-U_{x x}\right)}{2 \lambda_{i}-U_{x y}}=A_{i} c_{i}$,
$(i=1,2,3,4)$.

To get the values of constants $A_{i}$ and $\beta_{i}$. Let $\xi_{0}, \eta_{0}, \dot{\xi}_{0}$ and $\dot{\eta}_{0}$ be the initial coordinates and components of velocity then Equations (17) and (18) give at $\mathrm{t}=0$

$$
\begin{gathered}
\xi_{0}=A_{1}+A_{2}+A_{3}+A_{4} \\
\dot{\xi}_{0}=A_{1} \lambda_{1}+A_{2} \lambda_{2}+A_{3} \lambda_{3}+A_{4} \lambda_{4} \\
\eta_{0}=c_{1}\left(A_{1}+A_{2}\right)+i c\left(A_{3}+A_{4}\right) \\
\dot{\eta}_{0}=c_{1} \lambda_{1}\left(A_{1}+A_{2}\right)+i s c\left(A_{3}+A_{4}\right)
\end{gathered}
$$

By putting $A_{1}=A_{2}=0$ to eliminate unstable frequencies $\lambda_{1}$ and $\lambda_{2}$, then the Equations (19) through (22) become

$$
\begin{gathered}
\xi_{0}=A_{3}+A_{4} \\
\eta_{0}=i c\left(A_{3}+A_{4}\right) \\
\dot{\eta}_{0}=i s c\left(A_{3}+A_{4}\right) \\
\dot{\xi}_{0}=A_{3} \lambda_{3}+A_{4} \lambda_{4} \\
A_{3}=\frac{\xi_{0}}{2}-\frac{\eta_{0} i}{2 c} \\
A_{4}=\frac{\xi_{0}}{2}+\frac{\eta_{0} i}{2 c}
\end{gathered}
$$

Then Equations (17) and (18) become

$$
\xi=\frac{1}{2} \xi_{0}\left(\mathrm{e}^{-i s t}+\mathrm{e}^{i s t}\right)+\frac{\left(\eta_{0} i\right)\left(\mathrm{e}^{i s t}-\mathrm{e}^{-i s t}\right)}{2 c}=\frac{\eta_{0} \sin (s t)}{c}+\xi_{0} \cos (s t)
$$




$$
\eta=\frac{1}{2}\left(c \xi_{0}\right)\left(\mathrm{e}^{i s t}-\mathrm{e}^{-i s t}\right)+\frac{1}{2} i \eta_{0}\left(\mathrm{e}^{-i s t}+\mathrm{e}^{i s t}\right)=\eta_{0} \cos (s t)-c \xi_{0} \sin (s t)
$$

\section{Lissajoues Orbits at Collinear Points for Earth-Moon System}

To get the Lissajoues orbits around collinear points under the effect of oblateness, put $\eta_{0}=0$, in Equations (29) and (30) then $A_{3}=A_{4}=A_{\xi}=\frac{1}{2} \xi_{0}$

$$
\begin{array}{r}
\xi[t]=A_{\xi} \cos (s t+\varphi) \\
\eta[t]=-A_{\xi} \sin (s t+\varphi) \\
\zeta[t]=A_{\zeta} \cos (\sigma+t v)
\end{array}
$$

where, $\varphi$ and $\sigma$ are the phase angle.

Equations (31), (32) and (33) are used to determine the halo and Lissajoues orbits around any collinear libration points under the effect of oblateness.

\section{Phase Spaces at Libration Points}

To get the periodic orbits about the libration points the following technique will be used. This technique depends on the solution of the system of Equations (1), (2) and (3) taken into account the location of libration point as initial values, it is needed to reduce the order of the differential equations system as follows, let

$$
\begin{gathered}
\dot{x}(t)=u(t) \\
\dot{y}(t)=v(t) \\
\dot{z}(t)=w(t) \\
\dot{u}(t)=n^{2} x(t)+2 n v(t)-\frac{(1-\mu)(\mu+x(t))}{R_{1}(t)^{3}} \\
-\frac{\mu(\mu+x(t)-1)}{R_{2}(t)^{3}}-\frac{A_{1}(\mu+1)(\mu+x(t))}{R_{1}(t)^{5}} \\
\dot{v}(t)=n^{2} y(t)-2 n u(t)-\frac{(1-\mu) y(t)}{R_{1}(t)^{3}}-\frac{\mu y(t)}{R_{2}(t)^{3}} \\
-\frac{A_{1}(\mu+1)(\mu+x(t))}{R_{1}(t)^{5}} \\
\dot{w}(t)=-\frac{(1-\mu) z(t)}{R_{1}(t)^{3}}-\frac{\mu z(t)}{R_{2}(t)^{3}}
\end{gathered}
$$

\section{Results and Discussion}

The system of Equations (31), (32) and (33) are used to generate the periodic orbits around libration point $L_{2}$ which Figure 2 displays Lyabnuov planer orbit. Figure 3 and Figure 4 demonstrate the Lissajoues orbits around $L_{2}$ for the Earth-Moon system. The system of differential Equations (34) through (39) can be solved numerically, a code with MATHEMATICA was constructed to solve 


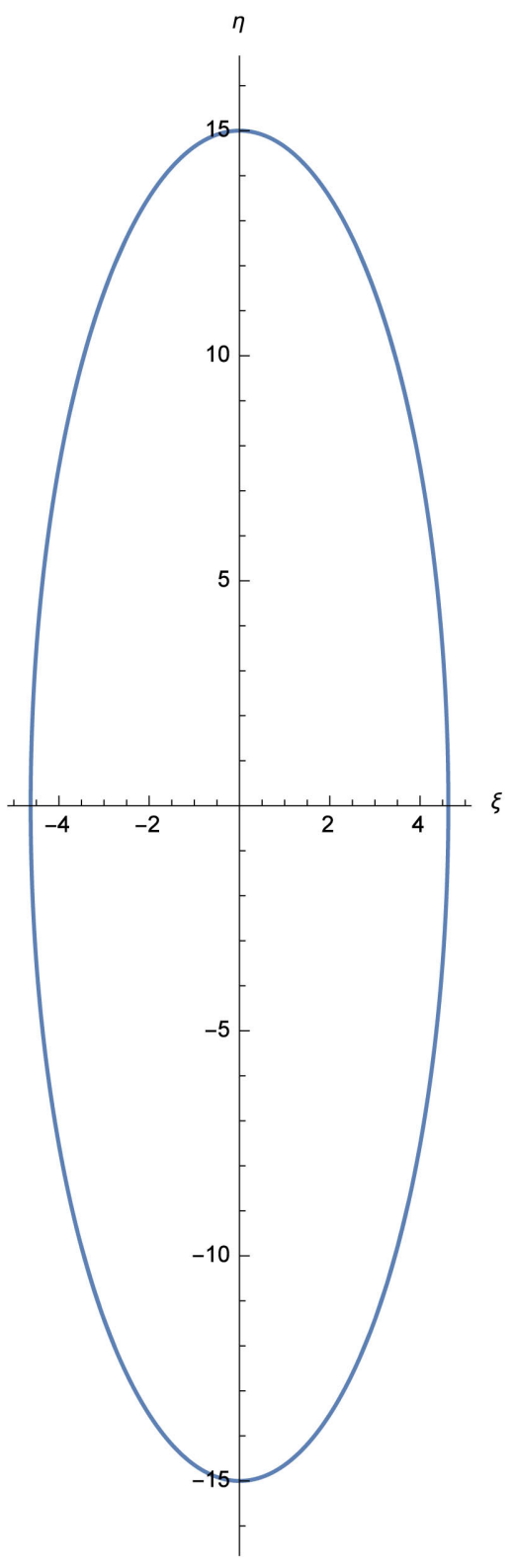

Figure 2. Lyapunov orbit around L2.

this system using Implist Runge Kutta method. Figure 5 displays the phase space for the motion near L1, also Figure 6 and Figure 7 display the phase space near L2 and L3 for the Earth-Moon-spacecraft system. Table 1 displays the collinear libration points and the eigen values at each point when the coefficient of oblateness $A=0$, and $A=0.001$.

\section{Conclusion}

Periodic orbits around collinear points in the restricted three-body problem have been studied under the effect of oblateness due to the bigger primary which enables the uses of these effects in the space missions. The obtained Lissijous orbit is one aim of the maneuvers through the path of any space craft. 


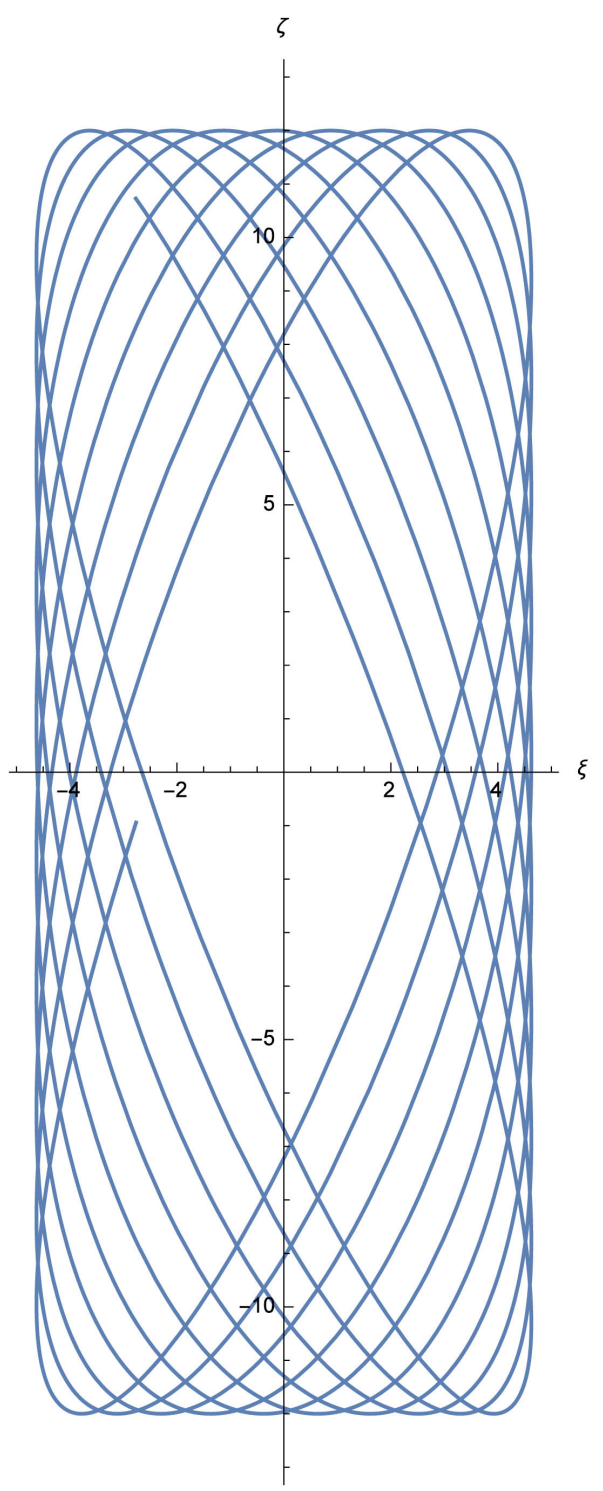

Figure 3. Lissajoues orbit $(x, z)$.

Table 1. Earth-Moon libration points at $\mu=0.0121505816$ and components of charcterstic roots.

\begin{tabular}{cccc}
\hline$A$ & $\mathrm{~L} 1$ & $\lambda_{1,2}$ & $\lambda_{3,4}$ \\
\hline 0 & 0.837659 & \pm 2.93205 & $\pm 2.33438 \mathrm{i}$ \\
0.001 & 0.837799 & \pm 2.95411 & $\pm 2.34699 \mathrm{i}$ \\
$A$ & $\mathrm{~L} 2$ & $\lambda_{1,2}$ & $\lambda_{3,4}$ \\
0 & 1.1551 & \pm 2.17167 & $\pm 1.87026 \mathrm{i}$ \\
0.001 & 1.00501 & \pm 2.2905. & $\pm 1.93957 \mathrm{i}$ \\
$A$ & $\mathrm{~L} 3$ & $\lambda_{1,2}$ & $\lambda_{3,4}$ \\
0 & -1.1551 & \pm 0.276486 & $\pm 1.02477 \mathrm{i}$ \\
0.001 & -1.15476 & \pm 0.278137 & $\pm 1.02144 \mathrm{i}$ \\
\hline
\end{tabular}




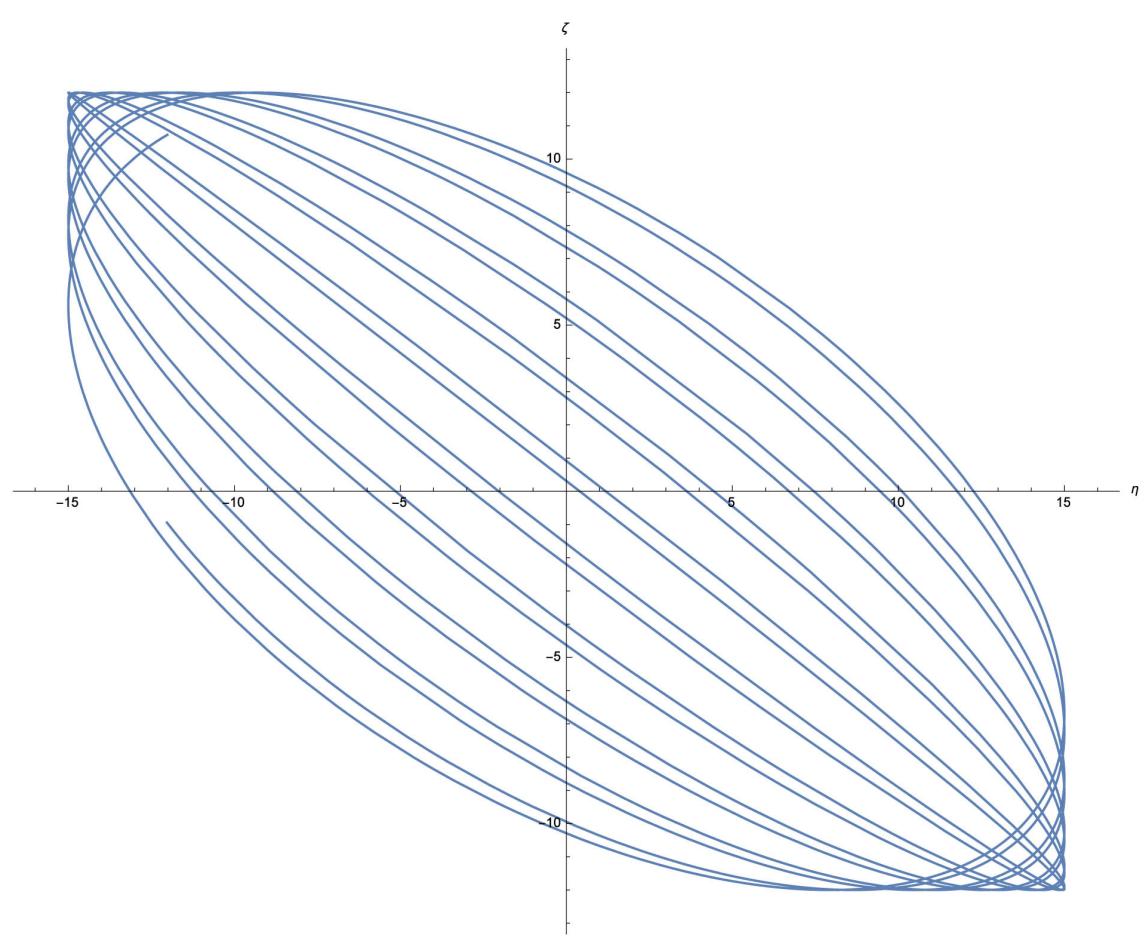

Figure 4. Lissajoues orbit $(y, z)$.

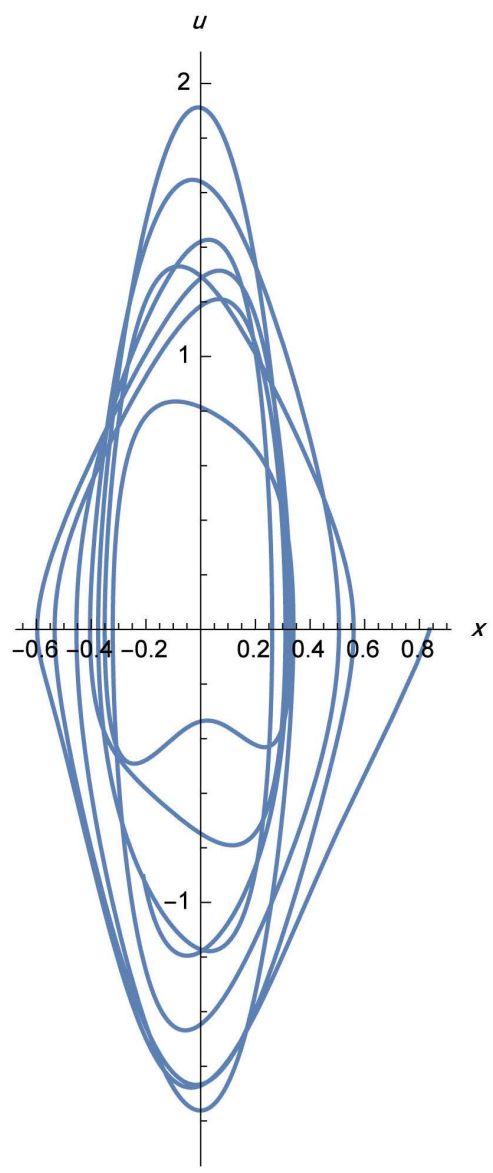

Figure 5. Phase space at L1. 


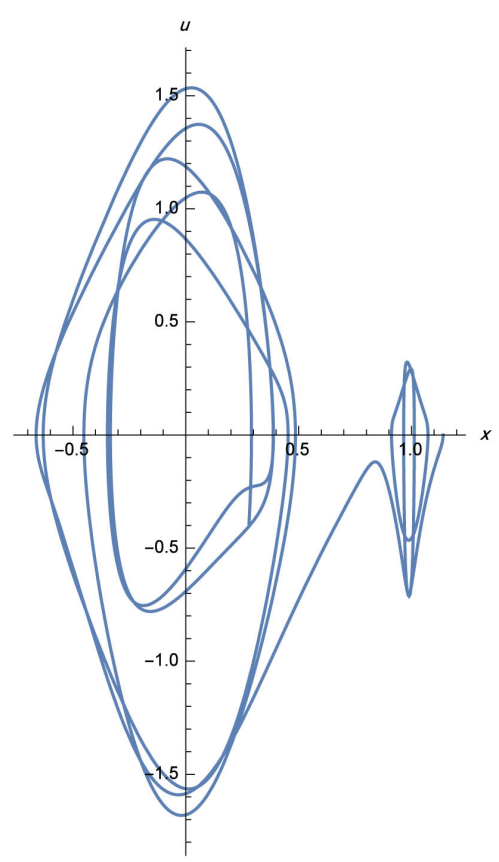

Figure 6. Phase space at L2.

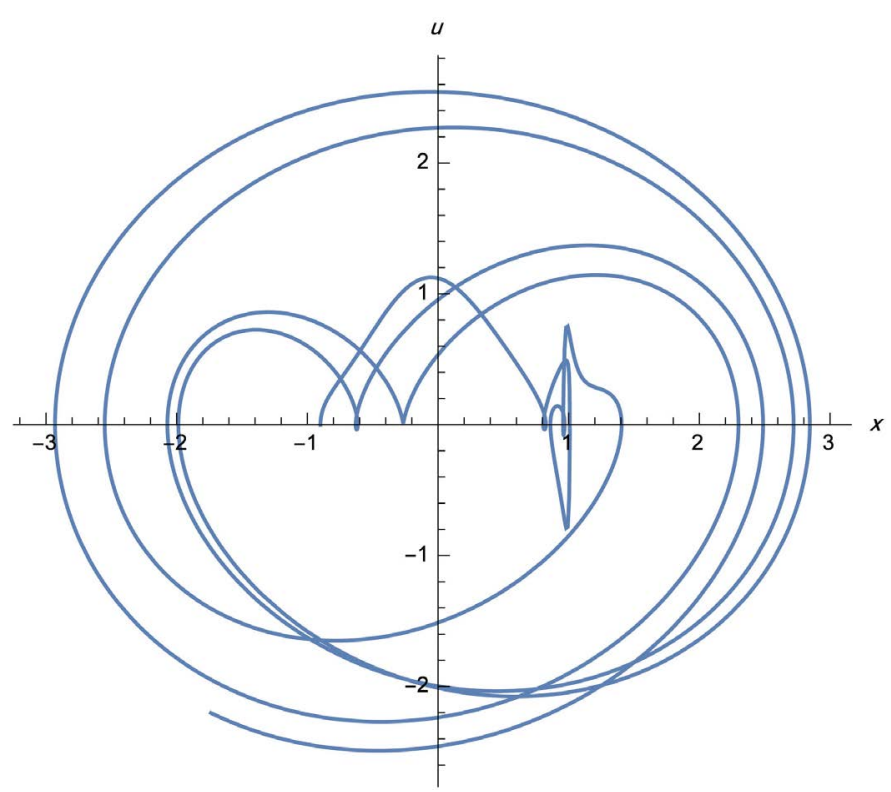

Figure 7. Phase space at L3.

\section{Acknowledgements}

The authors are grateful for the referees and the editor for their constructive suggestions.

\section{References}

[1] Szebehely, V.G. (1967) Theory of Orbits: The Restricted Problem of Three-Bodies. Academic Press Inc., New York.

[2] Curtis, H.D. (2009) Orbital Mechanics for Engineering Students. 2nd Edition, El- 
sevier Ltd., Amsterdam.

[3] Sharma, R.K. and Subba Rao, P.V. (1976) Stationary Solutions and Their Characteristic Exponents in the Restricted Three-Body Problem When the More Massive Primary Is an Oblate Spheroid. Celestial Mechanics, 13, 137-149.

https://doi.org/10.1007/BF01232721

[4] Ibrahim, A.H., Ismail, M.N. and Khalil, I.K.H. (2016) Studying the Libration Points of the Sun-Earth-Moon System. International Journal of Scientific and Engineering Research, 7, No. 10.

[5] Ismail, M.N., Khalil, I.K.H. and Ibrahim, A.H. (2016) The Effect of Solar Radiation Pressure on the Libration Points of the Restricted Four-Body Problem. Global Journal of Advanced Research, 3, 901-906.

[6] Archambeau, G., Augros, P. and Trélat, E. (2008) Eight Lissajous Orbits in the Earth-Moon System.

[7] Celletti, A., Pucacco, G. and Stella, D. (2015) Lissajous and Halo Orbits in the Restricted Three-Body Problem. Journal of Nonlinear Science, 25, 343-370.

https://doi.org/10.1007/s00332-015-9232-2

[8] Abouelmagd, E.I. and El-Shaboury, S.M. (2012) Periodic orbits under Combined Effects of Oblateness and Radiation in the Restricted Problem of Three Bodies. Astrophysics and Space Science, 341, 331-341. 